\title{
EVIDÊNCIAS DE HIPERFLUXO PORTAL NO PÓS-OPERATÓRIO DE TRANSPLANTES HEPÁTICOS*
}

\author{
Luciana Costa Silva ${ }^{1}$, Agnaldo Soares Lima ${ }^{2}$, Wilson Campos Tavares Júnior ${ }^{3}$, \\ Paulo Roberto Savassi Rocha ${ }^{4}$
}

Resumo OBJETIVO: A cirrose hepática causa alterações acentuadas na circulação esplâncnica. Com o objetivo de investigar as alterações hemodinâmicas na velocidade do fluxo da veia porta, realizamos estudos com ecoDoppler em pacientes com cirrose hepática em estágio final antes, durante e após transplante hepático ortotópico (THO). MATERIAIS E MÉTODOS: Cinqüenta e quatro pacientes submetidos a THO e eco-Doppler intraoperatório foram avaliados prospectivamente, entre janeiro de 2002 e julho de 2003. Dezessete pacientes foram excluídos devido a dados incompletos relacionados a dificuldades técnicas para obter medidas ou morte prematura. Os 37 pacientes incluídos na análise tinham cirrose hepática associada principalmente a infecção pelo vírus da hepatite $\mathbf{C}$ e doença alcoólica. Todos os pacientes foram submetidos ao eco-Doppler imediatamente antes do THO, no período intra-operatório após a reperfusão do enxerto e no primeiro e sétimo dias do pós-operatório. A velocidade média do fluxo foi medida no tronco, ramo direito e ramo esquerdo da veia porta. A análise estatística foi realizada utilizando-se o teste $t$ pareado e as diferenças foram consideradas significantes se $p<0,05$. RESULTADOS: A velocidade média observada no pré-THO foi de $16.0 \mathrm{~cm} / \mathrm{s}$. As medidas intra-operatórias, realizadas minutos após a reperfusão do enxerto, mostraram aumento na velocidade do fluxo da veia porta para $84.09 \mathrm{~cm} / \mathrm{s}$. A velocidade do fluxo da veia porta foi de $71,0 \mathrm{~cm} / \mathrm{s}$ e $58,5 \mathrm{~cm} / \mathrm{s}$ no primeiro e sétimo dias do pós-operatório, respectivamente. As velocidades obtidas no intra e pós-operatório foram significativamente maiores do que no pré-THO ( $p<0,001)$. No sétimo dia do pós-operatório, a velocidade média diminuiu significativamente em comparação aos valores do intraoperatório ( $p$ < 0.05). CONCLUSÃO: Nos transplantes hepáticos ocorre aumento significativo da velocidade média de fluxo da veia porta imediatamente após a reperfusão do enxerto em comparação aos valores préTHO. De maneira semelhante, após esse pico máximo parece ocorrer redução significativa e progressiva na velocidade média de fluxo, atingindo valores próximos da normalidade no sétimo dia do pós-operatório. Unitermos: Veia porta; Velocidade média; Transplante; Hemodinâmica.

Abstract Postoperative hyperflow in portal vein after liver transplantation.

OBJECTIVE: A marked impact on the splanchnic circulation is seen in liver cirrhosis. Doppler ultrasonography (DUS) was performed in a group of patients with end-stage liver cirrhosis before, during and after orthotopic liver transplantation (OLT) in order to investigate the hemodynamic changes in portal vein flow velocity. MATERIALS AND METHODS: Fifty-four patients submitted to OLT and intraoperative DUS were prospectively studied from January 2002 to July 2003. Seventeen patients were excluded because of missing data due to either technical difficulties to obtain the measurements or early patient death. All 37 patients included in the analysis had liver cirrhosis mainly associated with hepatitis $\mathrm{C}$ virus infection and alcoholic disease. Patients were submitted to DUS just before OLT, intraoperatively after graft reperfusion, and in the postoperative period (1st and 7th postoperative days). Mean flow velocity was measured at main, right and left portal veins. Statistical analysis was performed using paired $t$ test. Differences were significant when $\mathrm{p}<0.05$. RESULTS: The mean pre-OLT velocity in the portal vein was $16.0 \mathrm{~cm} / \mathrm{s}$. Intraoperative measurements obtained few minutes after graft reperfusion showed an increase in portal vein velocity to 84.09 $\mathrm{cm} / \mathrm{s}$. Portal flow velocity was $71.0 \mathrm{~cm} / \mathrm{s}$ and $58.5 \mathrm{~cm} / \mathrm{s}$ in the $1 \mathrm{st}$ and 7 th postoperative days, respectively. Intraoperative and postoperative mean flow velocity in the portal vein was significantly higher than in pre-OLT period $(\mathrm{p}<0.001)$. On the 7 th postoperative day, the mean flow velocity in the portal vein decreased significantly compared to intraoperative values $(p<0.05)$. CONCLUSION: In liver transplantation, there is a significantly increase in mean flow velocity in the portal vein immediately after graft reperfusion when compared to pre-OLT data. Likewise, after this initial peak flow there is a significant and progressive decrease in mean flow velocity in the portal vein, which tends to normalize on the 7th postoperative day. Key words: Portal vein; Mean velocity; Transplantation; Hemodynamics.

* Trabalho realizado no Serviço de Diagnóstico por Imagem do Hospital das Clínicas da Universidade Federal de Minas Gerais (HC-UFMG), Grupo de Transplantes do Instituto Alfa de Gastroenterologia do HC-UFMG, Belo Horizonte, MG.

1. Médica Assistente do Departamento de Radiologia e Diagnóstico por Imagem do HC-UFMG, Membro Titular do Colégio Brasileiro de Radiologia e Diagnóstico por Imagem (CBR).
2. Professor Adjunto do Departamento de Cirurgia da Faculdade de Medicina da UFMG (FM-UFMG), Coordenador do Grupo de Transplante do Instituto Alfa de Gastroenterologia.

3. Médico Residente do Departamento de Radiologia e Diagnóstico por Imagem do HC-UFMG.

4. Professor Titular do Departamento de Cirurgia da FMUFMG, Chefe do Instituto Alfa de Gastroenterologia.
Endereço para correspondência: Dra. Luciana Costa Silva. Rua Antonio Albuquerque, 877, ap. 503, Savassi. Belo Horizonte, MG, 30112-011. E-mail: Luciana.costa.silva@terra. com.br; wilsontavaresjrmd@yahoo.fr

Recebido para publicação em 30/7/2004. Aceito, após revisão, em 9/12/2004. 


\section{INTRODUÇÃO}

O transplante hepático tem-se provado um procedimento efetivo no tratamento de várias doenças hepáticas. Na maioria dos casos, ele é realizado em pacientes hepatopatas com hipertensão portal. Com o implante de um novo fígado, institui-se nova dinâmica do fluxo portal, não existindo mais o estado de hipertensão.

Existem, na literatura, relatos de alterações hemodinâmicas após transplante hepático ${ }^{(\mathbf{1})}$. Essas alterações se caracterizam, principalmente, por hiperfluxo portal. A função hepática imediata é muito sensível à congestão do enxerto, sendo dependente não só do afluxo portal, mas também do efluxo venoso hepático. A influência do excessivo fluxo portal tem sido associada à disfunção hepática, principalmente em casos de partições, como "splits" e transplante hepático doador-vivo. Esta disfunção é chamada de "small-for-size syndrome"(2).

Conduzimos o estudo com o objetivo de avaliar a velocidade média na veia porta através de eco-Doppler, antes, durante e após transplantes hepáticos.

\section{MATERIAIS E MÉTODOS}

Foi realizado estudo prospectivo com 37 pacientes submetidos a transplante hepático em nossa instituição, no período de janeiro de 2002 a julho de 2003. Todos os pacientes foram estudados por meio de eco-Doppler de vasos hepáticos à admissão para o transplante, durante o procedimento cirúrgico (após as anastomoses vasculares) e no primeiro e sétimo dias de pósoperatório. Realizaram-se medidas da velocidade média do tronco da veia porta e em seus ramos direito e esquerdo. Foram utilizados aparelhos das marcas ATL 3500 e Medison Gaia, com transdutores de 2,5 a $5,0 \mathrm{MHz}$ e 3,5 a $5,0 \mathrm{MHz}$, respectivamente. A análise estatística foi realizada utilizando o teste $t$ pareado, assumindo significância estatística com valor $p$ de 0,05 e intervalo de confiança (IC) de $95 \%$. Foram ainda analisados dados descritivos.

A idade média dos pacientes foi de 41,6 anos, com mediana de 44,0 anos (mínima de 1,0 ano, máxima de 69,0 anos, desviopadrão de 17,5 anos), sendo $37,8 \%$ do sexo feminino e $62,2 \%$ do sexo masculino. A maioria dos pacientes era portadora de hepatopatia etanólica (Tabela 1).

\section{RESULTADOS}

As medidas obtidas da velocidade média no tronco, ramos direito e esquerdo da

Tabela 1 Distribuição da etiologia da hepatopatia crônica nos pacientes estudados.

\begin{tabular}{|l|r|c|}
\hline Etiologia & $\mathrm{N}$ & Porcentagem \\
\hline Etanólica & 5 & $13,5 \%$ \\
Auto-imune & 4 & $10,8 \%$ \\
Colangite esclerosante & & \\
primária & 4 & $10,8 \%$ \\
Criptogênica & 4 & $10,8 \%$ \\
Atresia de vias biliares & 3 & $8,1 \%$ \\
Cirrose biliar primária & 3 & $8,1 \%$ \\
Vírus C & 10 & $27,0 \%$ \\
Budd-Chiari & 1 & $2,7 \%$ \\
Deficiência de alfa-1 & 1 & $2,7 \%$ \\
antitripsina & 1 & $2,7 \%$ \\
Vírus B & 1 & $2,7 \%$ \\
Vírus C e etanólica & 37 & $100,0 \%$ \\
\hline Total &
\end{tabular}

veia porta estão relacionadas nas Tabelas $2,3$ e 4$)$.

Quando comparamos as velocidades médias obtidas no tronco da veia porta no pré-operatório imediato e no peroperatório (logo após reperfusão), notamos diferença com significância estatística (valor $\mathrm{p}$ $=0,01$, IC de 95\%). Valores semelhantes foram observados também quando comparamos as velocidades médias do pré-operatório e dos primeiro e sétimo dias de pósoperatório (valor $\mathrm{p}<0,01$, IC de 95\%).

No seguimento, nenhum dos pacientes estudados apresentou complicações relacionadas à veia porta (Figuras $1 \mathrm{a} 4$ ).

\section{DISCUSSÃO}

O transplante hepático ortotópico é a modalidade ideal de tratamento para várias hepatopatias crônicas fibrosantes e em casos de hepatite fulminante.

O ultra-som com Doppler é o exame inicial na avaliação desses pacientes, tanto no pré quanto no pós-operatório, e permite a detecção de complicações vasculares variadas, incluindo trombose e estenose

Tabela 2 Comparação da velocidade média no tronco da veia porta no pré-operatório, no peroperatório e nos primeiro (1음ㅇ) e sétimo ( $7^{\circ}$ DPO) dias de pós-operatório.

\begin{tabular}{|l|c|c|c|c|c|}
\hline Tronco da veia porta & $\begin{array}{c}\text { Velocidade } \\
\text { mínima }\end{array}$ & $\begin{array}{c}\text { Velocidade } \\
\text { máxima }\end{array}$ & Média & Mediana & Desvio-padrão \\
\hline Pré-operatório & $-0,76$ & 35,00 & 16,00 & 13,20 & 8,60 \\
Peroperatório & 14,70 & 202,26 & 84,09 & 77,35 & 38,60 \\
1o DPO & 13,00 & 156,50 & 71,00 & 67,05 & 38,60 \\
7o DPO & 21,00 & 142,13 & 58,46 & 53,55 & 28,10 \\
\hline
\end{tabular}

Tabela 3 Comparação da velocidade média no ramo direito da veia porta no pré-operatório, no perope-

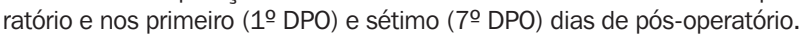

\begin{tabular}{|l|c|c|c|c|c|}
\hline $\begin{array}{l}\text { Ramo direito } \\
\text { da veia porta }\end{array}$ & $\begin{array}{c}\text { Velocidade } \\
\text { mínima }\end{array}$ & $\begin{array}{c}\text { Velocidade } \\
\text { máxima }\end{array}$ & Média & Mediana & Desvio-padrão \\
\hline Pré-operatório & $-9,00$ & 27,50 & 11,84 & 9,53 & 7,88 \\
Peroperatório & 27,30 & 161,00 & 84,07 & 73,41 & 43,89 \\
1o DPO & 8,75 & 138,40 & 56,09 & 47,90 & 34,39 \\
7o DPO & 19,00 & 87,80 & 48,05 & 48,60 & 21,23 \\
\hline
\end{tabular}

Tabela 4 Comparação da velocidade média no ramo esquerdo da veia porta no pré-operatório, no pero-

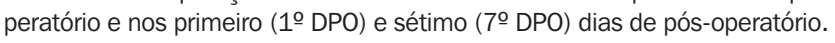

\begin{tabular}{|l|r|r|r|r|c|}
\hline $\begin{array}{l}\text { Ramo esquerdo } \\
\text { da veia porta }\end{array}$ & $\begin{array}{c}\text { Velocidade } \\
\text { mínima }\end{array}$ & $\begin{array}{c}\text { Velocidade } \\
\text { máxima }\end{array}$ & Média & Mediana & Desvio-padrão \\
\hline Pré-operatório & $-10,30$ & 34,00 & 11,07 & 8,50 & 9,33 \\
Peroperatório & 7,70 & 116,50 & 37,90 & 30,50 & 27,48 \\
1oDㅇ & 9,70 & 69,13 & 33,61 & 31,50 & 15,43 \\
7oㅡㅇㅡ & 10,97 & 45,00 & 25,42 & 21,00 & 11,00 \\
\hline
\end{tabular}




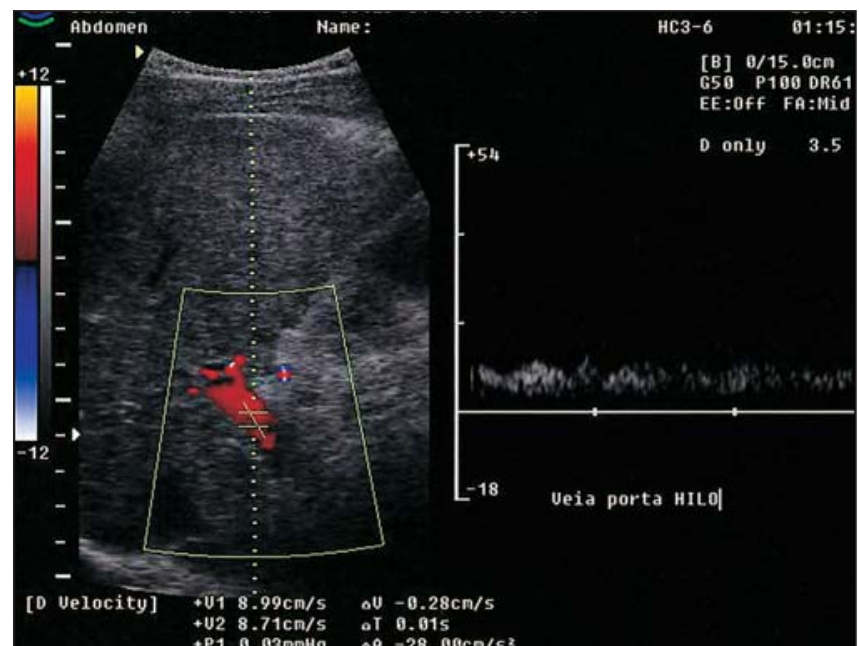

Figura 1. Eco-Doppler do tronco da veia porta no pré-operatório imediato, em que se observa velocidade de $28 \mathrm{~cm} / \mathrm{s}$.

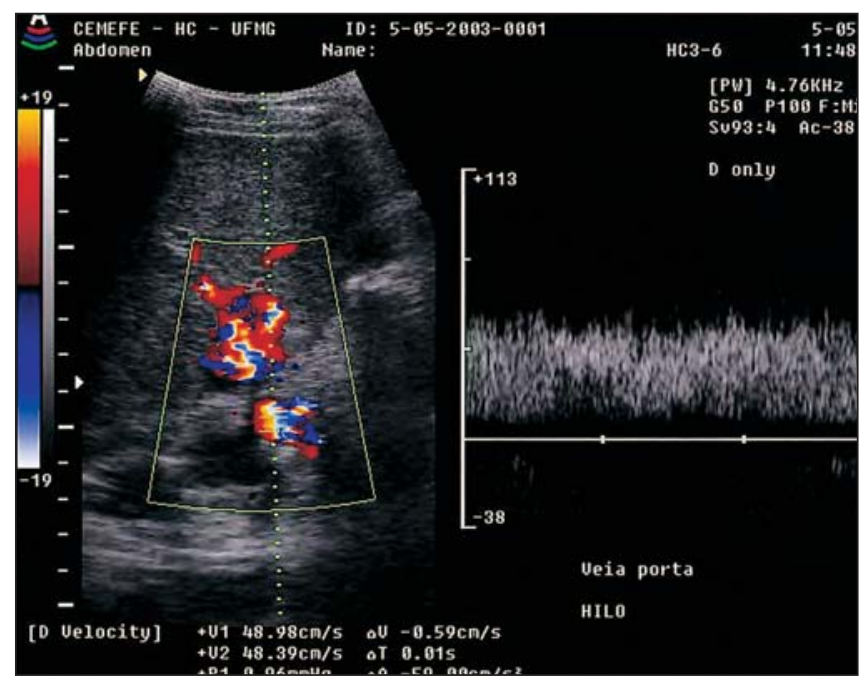

Figura 3. Eco-Doppler do tronco da veia porta no sétimo dia de pós-operatório, em que a velocidade era de $59 \mathrm{~cm} / \mathrm{s}$.

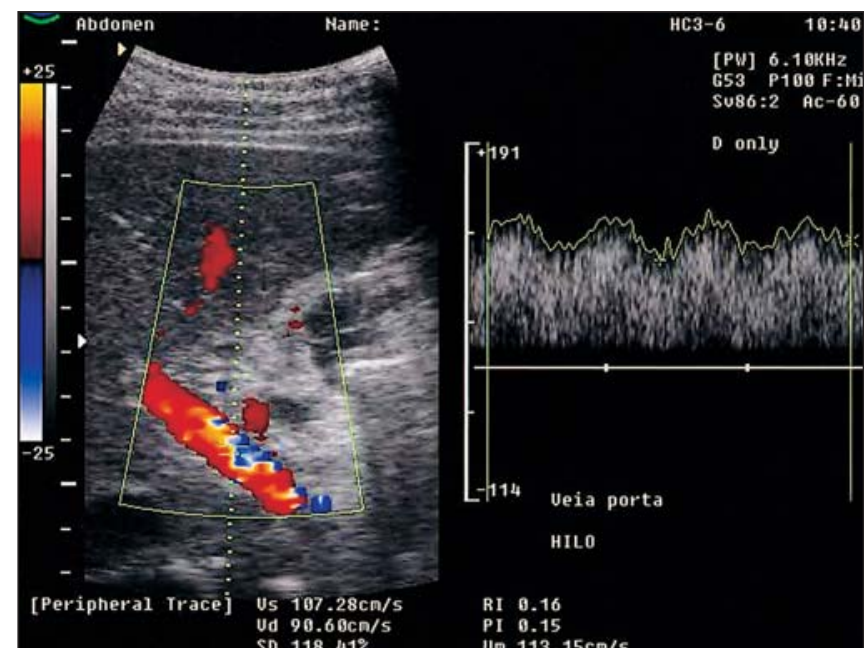

Figura 2. Eco-Doppler do tronco da veia porta no primeiro dia de pós-operatório, em que se observa velocidade de $113,15 \mathrm{~cm} / \mathrm{s}$.

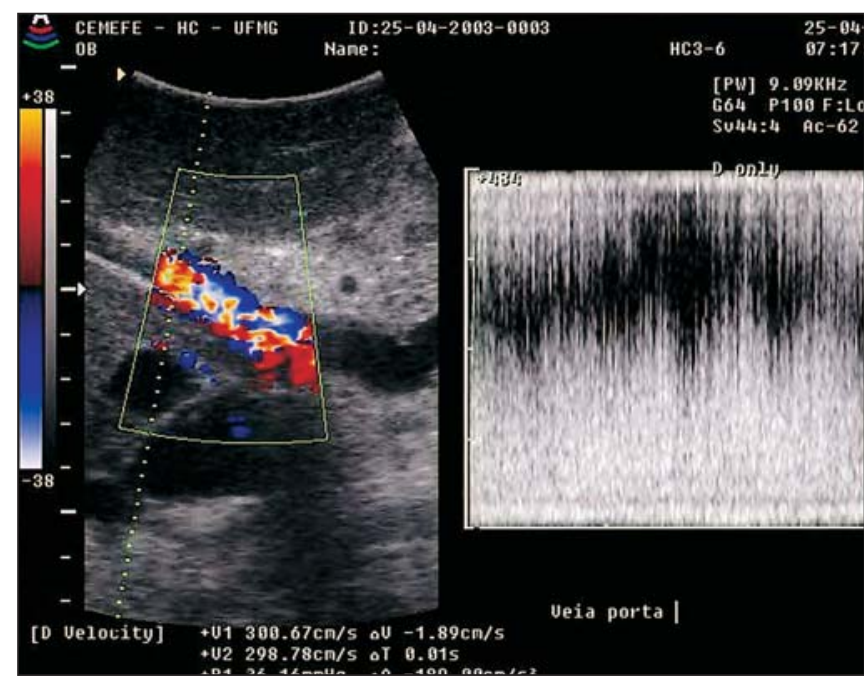

Figura 4. Eco-Doppler durante o procedimento cirúrgico (após as anastomoses vasculares), em que se nota velocidade de $189 \mathrm{~cm} / \mathrm{s}$. da artéria hepática, veia porta e veia cava inferior, além de pseudo-aneurismas da artéria hepática e estenoses do tronco celíaco. Há relatos da importância de se realizar estudo dopplerfluxométrico também no peroperatório ${ }^{(3)}$.

Conduzimos este estudo com a finalidade de avaliar a velocidade média da veia porta no pré-operatório, no peroperatório (logo após a reperfusão hepática) e no pósoperatório.

Segundo vários autores, o padrão de onda da veia porta em indivíduos normais é de um fluxo contínuo, hepatopetal, com discretas variações da velocidade induzidas pela respiração. Ao estudo espectral, con- sideram-se normais velocidades médias variando entre 12 e $30 \mathrm{~cm} / \mathrm{s}^{(4)}$.

Caracteriza-se estenose da veia porta quando existe "aliasing" focal ao Doppler colorido, com fluxo turbulento, e a velocidade média está aumentada em três a quatro vezes em relação ao segmento préestenótico $^{(\mathbf{5 , 6})}$.

Nosso estudo verificou que existe aumento significativo da velocidade média da veia porta, logo após a reperfusão do enxerto e no pós-operatório precoce, em relação aos valores considerados normais para indivíduos sadios. Este aumento situa-se, em média, pelo menos quatro vezes maior que o valor esperado para indi- víduos sadios $(20,0 \mathrm{~cm} / \mathrm{s})$ e pelo menos cinco vezes a velocidade média pré-transplante $(16,0 \mathrm{~cm} / \mathrm{s})$, com significância estatística $(\mathrm{p}<0,01)$.

Pacientes hepatopatas com cirrose avançada apresentam um desarranjo hemodinâmico sistêmico e no leito esplâncnico, que é caracterizado por um estado circulatório hipercinético ${ }^{(7)}$. Supõe-se que o aumento da velocidade na veia porta está relacionado à manutenção das alterações no leito vascular esplâncnico previamente existentes, com ausência de regulação do fluxo portal para o fígado transplantado. Assim, estabelece-se um estado de hiperfluxo portal. 
Alguns autores consideram este hiperfluxo causa de injúria hepatocelular ${ }^{(8)}$. Esta condição é melhor observada em transplantes intervivos e partições, os chamados "splits", em que a relação enxerto e peso do paciente é inadequada (enxerto com menos de $0,8 \%$ do peso do paciente) ${ }^{(7)}$. "Small-for-size" syndrome, definida como disfunção hepática precoce relacionada a enxertos pequenos em relação ao peso do receptor, possivelmente está relacionada a este estado de hiperfluxo portal $^{(9)}$.

Postula-se, também, a teoria da existência de uma compensação arterial ao hiperfluxo portal, representada por redução do fluxo arterial. Esta resposta "tampão" da artéria hepática pode ser fator predisponente à trombose $\operatorname{arterial}^{(\mathbf{1 0})}$.

\section{CONCLUSÃO}

A velocidade média na veia porta aumenta significativamente em pacientes transplantados hepáticos, quando comparada com a velocidade observada em indivíduos sadios e no pré-operatório de transplantes. Este aumento reflete um estado de hiperfluxo portal e estava presente em todos os pacientes estudados.

No presente estudo não houve complicações relacionadas à veia porta. Portanto, supõe-se que o aumento da velocidade portal, ao eco-Doppler, na população estudada, não deve ser considerado indicador de complicação vascular. Não se deve, então, erroneamente, caracterizar a presença de velocidade média aumentada na veia porta como estenose da anastomose, exceto se houver grande diferença em relação ao segmento pré-anastomótico.

\section{REFERÊNCIAS}

1. Huang TL, Cheng YF, Chen TY, et al. Portal hemodynamics in living-related liver transplantation: quantitative measurement by Doppler ultrasound. Transplant Proc 1988;30:3186-7.

2. Gondolesi GE, Florman S, Matsumoto C, et al. Venous hemodynamics in living donor right lobe liver transplantation. Liver Transpl 2002;8:80913.

3. Cheng YF, Huang TL, Chen CL, et al. Intraoperative Doppler ultrasound in liver transplantation. Clin Transplant 1998;12:292-9.

4 Shaw A, Sidhu PS. Ultrasound assessment of the liver transplant candidate. In: Sidhu PS, Baxter GM, eds. Ultrasound of abdominal transplantation. Stuttgart, Germany: Thieme, 2002;76-89.

5. Nghiem HV, Tran K, Winter TC III, et al. Imaging of complications in liver transplantation. RadioGraphics 1996;16:825-40.

6. Crossin JD, Muradali D, Wilson SR. US of liver transplants: normal and abnormal. RadioGraphics 2003;23:1093-114.

7. Garcia-Valdecasas JC, Fuster J, Charco R, et al. Changes in portal vein flow after adult living-donor liver transplantation: does it influence postoperative liver function? Liver Transpl 2003;9:564-9.

8. Troisi R, Cammu G, Militerno G, et al. Modulation of portal graft inflow: a necessity in adult livingdonor liver transplantation? Ann Surg 2003;237: 429-36.

9. Ott R, Schuppan D, Tannapfel A, et al. Portal vein arterialisation as a technical option in liver transplantation: impact on function, regeneration, and morphology of the liver following hemihepatectomy in pigs. Liver Int 2003;23:54-62.

10. Kiuchi T, Tanaka K, Ito T, et al. Small-for-size graft in living donor liver transplantation: how far should we go? Liver Transpl 2003;9:S29-35. 